\title{
Pharmacology for Anaesthesia and Intensive Care
}

\author{
Tom E. Peck, Sue A. Hill (eds). Cambridge University Press, Cambridge, UK, 2008, \\ 3rd edition, Paperback, 378 pp, ISBN 978-0-521-70463-2
}

\author{
Wilfred Demajo, MD
}

Published online: 24 January 2009

(c) Canadian Anesthesiologists' Society 2009

The targeted readers for this book, namely, residents in anesthesia and resident intensivists, are synonymous with the targeted readers for the very successful, previous two editions. The book is presented in four sections: Basic principles of pharmacology, Core drugs in anesthetic practice, Cardiovascular drugs, and Other important drugs. The differences between this and the previous two editions include an expanded and rewritten mathematics chapter in the first section, Basic principles, making it one of the positive highlights of the book, and the addition of a chapter on intravenous fluids in the final section, Other important drugs.

With its especially informative and concentrated chapters on the subjects of pharmacology of drugs, medicinal chemistry, mathematics, and pharmacokinetics, Basic principles is undeniably the best section of the book. In my opinion, the chapters in this section are well worth the price of the book and would be of great benefit to residents in any medical specialty. It would be disappointing if residents, other than anesthesia residents, were to ignore this book because of its title. In fact, in his foreword, Dr. Richard Griffiths suggested that the title should be changed in future editions to capture the interests of a broader audience. I wholeheartedly agree.

The second section regarding Core drugs in anesthesia practice is arranged in the usual manner with chapters on the subjects of general anesthetics, local anesthetic drugs, muscle relaxants, and anticholinesterase drugs. The material in this section is presented in a deceptively concise format when, in fact, it comprises a significant amount of detailed information. In this section, the facts are presented as capsules of information and then gradually integrated into a broader knowledge of the subject matter, which should help residents correctly answer multiple-choice questions. This section includes well-established information while excluding speculative topics. However, I was surprised not to find one comment about the use of lipids in the management of local anesthetic toxicity.

The third section is devoted to the subject of cardiovascular drugs, while the focus of the chapters in the final section, Other important drugs, is on drugs that are important in the management of diseases associated with the central nervous system, infections, diabetes, coagulation, and etc. This last section includes a new chapter on intravenous fluids which could be improved. The new chapter adds little to the quality of the book in its present format, considering the increased use of starch-based fluids and the contentions over the use of the various types and quantities of fluids administered.

Overall, the book is well written and the information is very well presented, and it merits both the reputation and the audience it has garnered. I would consider this text to be worthwhile reading for residents of all specialties, as well as staff intensivists who wish to update themselves on the important bases of practice in critical care medicine.

W. Demajo, MD ( $₫)$

Toronto Western Hospital, Toronto, Canada

e-mail: dr.wilfred.demajo@uhn.on.ca 